\title{
THE LATTICE DYNAMICS OF COMPLETELY ENTANGLED STATES AND ITS APPLICATION TO COMMUNICATION SCHEMES
}

\author{
Daniel I. Fivel \\ Department of Physics \\ University of Maryland, College Park, MD 20742-4111
}

\begin{abstract}
It is shown that among the orthogonal sets of EPR (completely entangled) states there is a unique basis (up to equivalence) that is a also a perfectly resolved set of coherent states with respect to a pair of complementary observables. This basis defines a lattice phase space in which quadratic Hamiltonians constructed from the observables induce site-to-site hopping at discrete time intervals. When recently suggested communication schemes [1] are adapted to the lattice they are greatly enhanced, because the finite Heisenberg group structure allows dynamic generation of signal sequences using the quadratic Hamiltonians. We anticipate the possibility of interferometry by determining the relative phases between successive signals produced by the simplest Hamiltonians of this type, and we show that they exhibit a remarkable pattern determined by the number-theoretic Legendre symbol.
\end{abstract}

Presented at the conference on Fundamental Problems in Quantum Theory University of Maryland at Baltimore - June 1994 
Several proposals have been made recently [1, 2] for employing EPR states [3] in cryptographic and other communication schemes. Evidently we are no longer to regard these states as theoretical curiosities useful only for discussions of the foundations of quantum mechanics. In this paper we will develop the theory of EPR dynamics, showing that EPR states form a natural lattice with a finite Heisenberg group structure, and we will explore the physical and mathematical consequences of that structure.

For the purposes of our discussion the term EPR states will refer to the subset $\mathcal{E}$ of completely entangled states in a two-particle Hilbert space $\mathcal{H}$, where each one-particle Hilbert space $\mathcal{H}_{N}$ is of finite dimension $N$. It can then be shown[ [4] that all such states are of the form:

$$
|S\rangle=N^{-1 / 2} \sum_{j=1}^{N}|j, 1\rangle\left|j^{\mathcal{U}}, 2\right\rangle,
$$

where $j$ labels any one-particle basis, and $\mathcal{U}$ indicates any one particle antiunitary transformation of the state. Thus, e.g. the familiar Bohm state (the spin- 0 state of two spin- $1 / 2$ particles) is obtained in the case $N=2$ by choosing $\mathcal{U}$ to be the time-reversal transformation. Note that the antiunitarity of $\mathcal{U}$ results in $|S\rangle$ being independent of the choice of basis so that EPR states act like unitary scalars. Since every anti-unitary transformation can be obtained from one of them by applying a unitary transformation, we may select a fiducial EPR state and obtain all the others from it by applying a one-particle unitary operator $u$ to particle-2. Thus it makes sense to denote the set of EPR states for given $N$ by $|u\rangle$ as $u$ runs over the group $U_{N}$ of $N$ by $N$ unitary matrices. If $u, v$ are two such matrices we write:

$$
|u v\rangle=u|v\rangle
$$

in which $u$ on the right side acts on particle-2, and from (1) we have the elegant formula for the scalar product of two EPR states:

$$
\langle u \mid v\rangle=N^{-1} \operatorname{Tr}\left(u^{\dagger} v\right)
$$

When we speak of orthogonal $u$ 's it will be in the sense of (3). Because of the one-one correspondence we will now drop the ket notation and simply use $u$ to denote either an EPR state or a one-particle operator.

There are clearly $N^{2}$ linearly independent EPR states, so that they span the two particle hilbert space. However, linear combinations of EPR states 
are not in general EPR states so that they themselves form a Riemannian manifold - not a subspace - in the two particle space. Thus one produces new EPR states from old ones by multiplying $u$ 's rather than by adding them. This is the basis of the Bennett-Wiesner communication scheme[1] which works in the following way: Communicators Alice and Bob agree on a set of $N^{2}$ mutually orthogonal $u$ 's, say $u_{1}, \cdots, u_{N^{2}}$ with $u_{1}$ being the initial EPR state prepared by Alice. Any such set of $u_{j}$ 's will be called an EPR-basis. Particle-2 is sent to Bob who applies any one of the $N^{2}$ operators $u_{j} u_{1}^{-1}$. This has the effect of transforming the two-particle state into $u_{j}$, and he then returns particle-2 to Alice. Since she owns both particles she can ascertain which of the $N^{2}$ orthogonal states the system is in by means of a generalized Stern-Gerlach apparatus. Now it is important to recognize that Alice cannot unilaterally decide to change the initial state from $u_{1}$ to one of the other basis states e.g. $u_{2}$ without informing Bob. The reason is that $u_{j} u_{1}^{-1} u_{2}$ will not in general coincide (up to phase) with any one of the $N^{2} u_{j}$ 's. Moreover, even if Alice does not change the initial state, Bob is still restricted to a single application of one of the operators $u_{j} u_{1}^{-1}$, i.e. he cannot employ a dynamical process that might involve an arbitrary product of $u_{j}$ 's. The reason is the same.

Now let us observe that both of the limitations just described will be eliminated if we are able to choose the $N^{2} u_{j}$ 's in such a way that they are not only mutually orthogonal but also form the ray representation of a multiplicative group $\mathcal{G}$. Let us see that there is a choice, and (up to equivalence) a unique choice, of the set of $u_{j}$ 's that will have this property:

We begin by observing that the qualifier "ray" is essential in that there will in general be no non-trivial true representations. To see this observe first that $\mathcal{G}$ cannot be abelian, since with $N \times N$ matrices at most $N$ linearly independent ones can commute. Now suppose that $N=p$, where $p$ is prime. It is known [5] that the only groups of order $p^{2}$ are abelian, namely the cyclic group of order $p^{2}$ and the direct product of two cyclic groups, each of order $p$. The first possibility does not give us a non-abelian result even when we extend to a ray representation by allowing arbitrary phases. However the second one does. To see why one need only recall how the coordinate and momentum operators $X, P$ of a particle each generate abelian groups $e^{i \alpha X}, e^{i \beta P}$ that also commute with one another except for a phase multiple. This immediately suggests how to construct the solution in the case where $N$ is a prime and leads us to expect a finite Heisenberg group. It will turn 
out, in fact, that the Heisenberg group is the only solution for non-prime $N$ as well - provided that the EPR states are not composites of simpler EPR states. In composite cases the wave-function will factorize and the associated group will be the direct product of lower order heisenberg groups.

From the analogy with the $e^{i \alpha X}, e^{i \beta P}$ operators, let $\sigma, \tau$ be unitary operators in the $N$-dimensional particle-2 Hilbert space $\mathcal{H}_{N}^{(2)}$ satisfying:

$$
\sigma \tau=\omega \tau \sigma, \quad \omega=e^{2 \pi i / N} .
$$

These can be realized by introducing a basis $|j\rangle, j=0,1, \cdots(\bmod N)$ in $\mathcal{H}_{N}^{(2)}$ and letting

$$
\sigma|j\rangle=\omega^{j}|j\rangle, \quad \tau|j\rangle=|j+1\rangle .
$$

(Note that $\tau|N-1\rangle=|0\rangle)$. We now define the $N^{2}$ u-matrices of our EPR basis to be: $u(\mathbf{j})$ where $\mathbf{j}=(j, k)$ with $j, k=0,1, \cdots, N-1$ and

$$
u(\mathbf{j})=e^{-i \pi j k / N} \sigma^{j} \tau^{k} .
$$

With this choice of phases we have the simple group multiplication law:

$$
u(\mathbf{j}) u\left(\mathbf{j}^{\prime}\right)=e^{i \pi \mathbf{j} \times \mathbf{j}^{\prime} / N} u\left(\mathbf{j}+\mathbf{j}^{\prime}\right), \quad \mathbf{j} \times \mathbf{j}^{\prime} \equiv j k^{\prime}-k j^{\prime} .
$$

This is analogous to the quantum mechanical rule for multiplying operators of the form $u(\alpha, \beta) \equiv e^{i(\alpha X+\beta P)}$ and defines the finite Heisenberg group $\mathcal{G}_{N}$. Note that the phase factor on the right is such that the operators on the left commute whenever $\mathbf{j}, \mathbf{j}^{\prime}$ are linearly dependent, whence in particular $u(n \mathbf{j})=$ $(u(\mathbf{j}))^{n}$. The trace-orthonormality of the $u(\mathbf{j})^{\text {'s }}$ may be verified by direct computation. Note that the mutual orthogonality of the $u(\mathbf{j})$ 's follows from:

$$
\operatorname{Tr}(u(\mathbf{j}))=N \delta_{\mathbf{j}, \mathbf{o}}
$$

where $\mathbf{o}$ is the zero vector.

The Heisenberg group structure we have obtained shows that the states of the lattice are exactly analagous to the coherent states associated with the coordinate-momentum Heisenberg group with the but with discrete-valued conjugate observables $\sigma$ and $\tau$. We will therefore refer to the distinguished set we have constructed as a coherent EPR basis. Note that the usual coherent states do not enjoy the orthogonality relationship of the $N^{2}$ coherent EPR states. The fact that the coherent EPR basis states are in this sense 
completely resolved expresses the circumvention of the uncertainty principle in the non-invasive measurements performed on EPR states.

As we have noted, the great virtue of the group property of the coherent basis in the BW communication scheme is that an arbitrary sequence of $u$ 's from the basis can be applied, and an arbitrary element from the basis can be unilaterally chosen to be the initial state. This flexibility also makes possible the process we describe next, namely the dynamic generation of sequences at discrete time intervals through the action of a suitable Hamiltonian $H$. Such a Hamiltonian must have the property that the associated time evolution operator $U(t)$ obtained from the Schrödinger equation $i \partial U / \partial t=H U$ will transform elements of the basis into one another at designated times $t=\left\{t_{n}\right\}$. Thus we must require:

$$
U(t) u(\mathbf{j}) U^{\dagger}(t)=u\left(\mathbf{j}^{\prime}\right) .
$$

Replacing $\mathbf{j}$ with $n_{1} \mathbf{j}_{\mathbf{1}}+n_{2} \mathbf{j}_{\mathbf{2}}$ where $n_{1}, n_{2}$ are integers and using (7), one deduces that $\mathbf{j}^{\prime}$ must be related to $\mathbf{j}$ by a linear transformation under which the cross-product is invariant. Thus each $U$ must correspond to an element $\mathcal{M}$ of the group $S L_{2}(Z / N)$ consisting of two-by-two matrices with elements in the ring of integers $\bmod (N)$ and unit determinant $(\bmod N)$.

In order to simplify the discussion without affecting the physics we will from here on assume that $N$ is a prime $p$. The reason is that $Z / p$ will then be a field $Z_{p}$, and so we may do matrix manipulations just as with complex numbers, the condition for an inverse being simply that the determinant not be divisible by $p$. (No eigenvalues will have to be computed, so the fact that $Z_{p}$ is not algebraically closed will not cause difficulties.) Generalization to non-prime $N$ is straightforward.

Our problem now is to construct unitary operators $U_{\mathcal{M}}$ such that

$$
U_{\mathcal{M}} u(\mathbf{j}) U_{\mathcal{M}}^{\dagger}=u(\mathcal{M} \mathbf{j})
$$

with $\mathcal{M} \in S L_{2}\left(Z_{p}\right)$. One guesses the answer based on experience with $X, P$ in quantum mechanics, namely that quadratic forms in $X, P$ generate linear transformations of the operators. By the completeness of the $u(\mathbf{j})$ 's we know that $U_{\mathcal{M}}$ can be expressed as a linear combination of them. We are thus led to try linear combinations in which the coefficients are phases constructed by exponentiating quadratic forms $\tilde{\mathbf{j}} \mathcal{Q} \mathbf{j}$ in which $\mathcal{Q}$ is a two-by-two matrix and the tilde indicates a row vector. Indeed we will find that (10) can be 
implemented with:

$$
U_{\mathcal{M}}=\sum_{\mathbf{j}} e^{(\pi i / 2 p) \tilde{\mathbf{j}} \mathcal{Q}_{\mathcal{M}} \mathbf{j}} u(\mathbf{j}),
$$

or possibly a degenerate form of this in which the double sum reduces to a simple sum.

Let us first exploit the fact [6] that $S L_{2}\left(Z_{p}\right)$ is generated by the two elements $\mathcal{M}=\rho, \chi$ with:

$$
\rho=\left(\begin{array}{ll}
1 & 1 \\
0 & 1
\end{array}\right), \quad \chi=\left(\begin{array}{ll}
1 & 0 \\
1 & 1
\end{array}\right)
$$

One readily verifies that the corresponding $U_{\mathcal{M}}$ will be the degenerate forms of (11) alluded to. In fact, making use of the familiar character identitiy:

$$
\sum_{n=0}^{p-1} e^{2 \pi i n / p}=\delta_{n 0},
$$

where the Kronecker symbol is $\bmod (p)$, a straightforward calculation will verify the following formula for the $U_{\mathcal{M}}$ 's corresponding to the cyclic subgroups containing the two generators:

$$
\begin{gathered}
\mathcal{M}=\left(\begin{array}{cc}
1 & m^{-1} \\
0 & 1
\end{array}\right) \rightarrow U_{\mathcal{M}}=\sum_{j} e^{-\pi i m j^{2} / p} u(j, 0), \\
\mathcal{M}=\left(\begin{array}{ll}
1 & 0 \\
m^{-1} & 1
\end{array}\right) \rightarrow U_{\mathcal{M}}=\sum_{k} e^{\pi i m k^{2} / p} u(0, k) .
\end{gathered}
$$

Sums are from 0 to $p-1$. The operators $U_{\rho}, U_{\chi}$ corresponding to the group generators $\rho, \chi$ are obtained by setting $m=1$. (Caution: In going from the left to the right side of (14) the $m$ corresponding to $m^{-1}$ in $Z_{p}$ must first be written as an ordinary integer $\bmod (p)$. Thus e.g. if $p=5$ and $m^{-1}=3$ appears in the matrix on the left, its $Z_{5}$ inverse $m=1 / 3$ should be written as $m=2$ in the exponent on the right, not directly inserted as $m=1 / 3$.)

One can now, in principle, find a $U_{\mathcal{M}}$ for any $\mathcal{M}$ by writing it as a "word" in the letters $\rho, \chi$, replacing them with $U_{\rho}, U_{\chi}$, and multiplying out the $U$ 's. This would be unnecessarily tedious as the following formula indicates: Suppose that in (11) we put:

$$
\mathcal{Q}_{\mathcal{M}}=\left(\begin{array}{lr}
2 a & b \\
b & 2 c
\end{array}\right),
$$


with integers a,b,c and with the restrictions on the discriminant $\Delta \equiv b^{2}-4 a c$

$$
\text { (i) } \Delta \not \equiv 1(\bmod p), \quad(\text { ii }) \Delta \equiv 1(\bmod 4), \text { i.e. } b \text { odd. }
$$

Then it can be shown that $\mathcal{Q}_{\mathcal{M}}$ is related to $\mathcal{M}$ by a Cayley transform:

$$
\mathcal{M}=\frac{\nu \mathcal{Q}_{\mathcal{M}}+I}{\nu \mathcal{Q}_{\mathcal{M}}-I}, \quad \nu=\left(\begin{array}{cc}
0 & 1 \\
-1 & 0
\end{array}\right)
$$

where the computations in (15) are in $Z_{p}$. Note that condition $(i)$ on $\Delta$ insures that the inverse in (13) exists and $(i i)$ is found to be needed in the course of calculation to permit use of (13).

While we shall not make use of it in the present paper it is interesting to see from (15) how the analogue of the canonical structure of quantum mechanics is expressed in the finite lattice phase space of EPR states: If $\mathcal{R}$ is a unimodular matrix one verifies that $\mathcal{R}^{-1} \nu=\nu \tilde{\mathcal{R}}$ so that (14) continues to hold under the transformation:

$$
\mathcal{M} \rightarrow \mathcal{R}^{-1} \mathcal{M} \mathcal{R}, \quad \mathcal{Q}_{\mathcal{M}} \rightarrow \tilde{\mathcal{R}} \mathcal{Q}_{\mathcal{M}} \mathcal{R}
$$

Thus each $U_{\mathcal{M}}$ producing a "hopping" of EPR states from one lattice site to another will have a counterpart under the canonical transformation $\mathcal{R}$ which, as one sees from its relation to $\nu$, is a finite symplectic transformation of the lattice. Note that $\mathcal{R}$ preserves the discriminant and therefore the two conditions used in (15).

Having now developed techniques for implementing (10) let us next examine the simplest example of a dynamical process that will produce a sequence of EPR states, namely an analogue in the lattice phase space of free particle motion. We produce this by iterating the generator $\rho$ of the first cyclic group in (14), i.e. we define:

$$
U(t)=\left(U_{\rho}\right)^{2 t}, \quad t=0,1, \cdots
$$

An effective Hamiltonian $H_{\rho}$ can be associated with this by:

$$
U_{\rho}=e^{-i H_{\rho} / 2}
$$

the additional factor of 2 having been inserted anticipating a simplification below. Thus (17) will be the formal solution of the corresponding Schrödinger 
equation determined by this Hamiltonian. A formal solution, however is not good enough for our purposes - we require an explicit formula for $U(t)$. We note that

$$
\left(U_{\rho}\right)^{2 t}=e^{i \psi(t)} U_{\rho^{2 t}}, \quad \rho^{2 t}=\left(\begin{array}{ll}
1 & 2 t \\
0 & 1
\end{array}\right),
$$

and so, in view of (14), we do have an explicit formula except for the phase factor. Now it is true that one does not need to know this factor for the computation of (9), since the reciprocal appears in $U^{\dagger}$. Thus if we are only interested in application to the BW communication scheme we require nothing more. However, we wish to go further than merely identifying the signals, in particular we envisage the possibility of doing some kind of interferometry with them. This will require a knowledge of the phases in (19), and as we shall see, they have a truly remarkable structure.

Note first that from (8), taking traces on both sides of (19) will give:

$$
e^{i \psi(t)}=p^{-1} \operatorname{Tr}\left(\left(U_{\rho}\right)^{2 t}\right)
$$

The right side has the structure of a partition function and can be manipulated in the same way. Thus if one inserts the right side of the first equation in (14) for $U_{\rho}$ (with $m=1$ ) there will be a product of sums indexed by $j_{1}, j_{2}, \cdots, j_{2 t}$ containing $u\left(j_{1}+\cdots+j_{2 t}, 0\right)$ which has zero trace unless the sum of the $j$ 's is $0 \bmod (p)$. One can then use (13) to pick out this term using a standard trick and obtain:

$$
\operatorname{Tr}\left(\left(U_{\rho}\right)^{2 t}\right)=\sum_{n}(F(n))^{2 t},
$$

and a completion of the square gives:

$$
F(n)=\sum_{j} e^{-i \pi j^{2} / p} e^{2 \pi i n j / p}=S(-2 p) e^{i \pi n^{2} / p} . \quad S(p)=\sum_{n} e^{2 \pi i n^{2} / p} .
$$

Thus

$$
e^{i \psi(t)}=p^{-1}(S(-2 p))^{2 t} S(p / t) .
$$

The function $S(p)$ is known as a Gauss sum and it is fundamental in the solution of quadratic diophantine equations. Let us examine the expression we have obtained a little more closely using the known exact expression[7] for $S(p / t)$ for an arbitrary odd prime $p$ namely:

$$
S(p / t)=\left(\frac{t}{p}\right) \sqrt{\left(\frac{-1}{p}\right) p}
$$


where the Legendre symbol is defined by:

$$
\left(\frac{t}{p}\right)=\left\{\begin{array}{l}
+1 \text { if } t \equiv \text { a square } \bmod p \\
-1 \text { if } t \not \equiv \text { a square } \bmod p
\end{array}\right.
$$

Observe that if an arbitrary constant is added to the Hamiltonian in (18), $U_{\rho}$ will be multiplied by a phase which will in turn produce a phase to the $2 t$ 'th power in (23). Hence the argument of the factor $(S(-2 p))^{2 t}$ can be "gauged" away along with the $t$ independent phase $\sqrt{(-1 / p)}$ in $(24)$. Since we know that the left side of (23) is unimodular it follows that the modulus of $S(-2 p)$ must be just $\sqrt{p}$. Thus up to gauge we have established the extremely simple fact that:

$$
e^{i \psi(t)}=\left(\frac{t}{p}\right)
$$

i.e. the time-evolving phase of the "free" EPR state follows a pattern of +1 's and -1 's in a manner with basic number theoretic significance. Just how simple this result is may be appreciated as follows: The notion of "sign" in the usual sense does not exist in the field $Z_{p}$, but, if for real numbers we definie a positive number as one that is the square of something while a negative number is one that isn't, then the notion generalizes to $Z_{p}$ as the Legendre symbol. It can also be shown that that for $p>2$ there are just as many squares (quadratic residues) as non-squares. Thus we have obtained the pleasing result that the analoge of free particle motion in the EPR lattice is characterized by a wave function with the analogue of a sign alternating phase! The computation of the Legendre symbol is facilitated by its wellknown factorization law which reduces it to a product of Legendre symbols whose upper members are the prime factors of $t$. These in turn obey the celebrated and profound Gauss law of quadratic reciprocity relating $\left(\frac{q}{p}\right)$ to $\left(\frac{p}{q}\right)$. It is clear from the above discussion that when we come to investigate and classify more general lattice Hamiltonians we will encounter generalized gaussian sums (theta-series) [6, 7] and will have to invoke the general theory of quadratic diophantine equations. It thus appears that we have just scratched the surface of fruitful connections between the lattice dynamics of EPR states and one of the richest areas of contemporary mathematics. For example it will be of utmost interest to ascertain the quantum mechanical significance of Gaussian quadratic reciprocity. 
I should like to acknowledge useful conversations with L. Washington and A. Dragt.

\section{References}

[1] Bennett, C. H. and S.J. Wiesner,1992, Phys. Rev. Let. 69, 2881.

[2] Bennett, C. H. G. Brassard, C. Crêpeau, R. Josza, A. Peres, and W. Wooters, 1993, Phys. Rev. Let. 70, 1895.

[3] Einstein, A. B. Podolsky, and N. Rosen, 1935 Phys. Rev. 47,777

[4] Fivel, D. I. 1994 Preprint UMD-PP-132, hep-th/9404178

[5] Hall, M. 1959, "Theory of Groups", p. 51, (MacMillan).

[6] Hua Loo Keng, 1982 "Introduction to Number Theory", p.367, (SpringerBerlin).

[7] Schoeneberg, B. 1974, "Eliptic Modular Functions", p.218, (SpringerBerlin) . 